\title{
Exosomes in Cardiovascular Diseases and Treatment: Experimental and Clinical Aspects
}

\author{
Jing Wang ${ }^{1} \cdot$ Chenglin Zhao $^{1} \cdot$ Junjie Xiao $^{1}$
}

Received: 19 December 2018 / Accepted: 21 December 2018 / Published online: 7 January 2019

(C) Springer Science+Business Media, LLC, part of Springer Nature 2019

Cardiovascular diseases (CVDs) are major health problem worldwide, which remain the leading causes of mortality globally. The incidence of CVDs continues to increase these days, leading to a growing epidemic which constitutes a serious financial burden for society [1-3]. Exosomes are smallsized membrane-surrounded structures that can be released from different types of cells, such as cardiomyocytes, endothelial cells, fibroblasts, stem cells, and immune cells. The intercellular communications that mediated by exosomes play a pivotal role in a variety of cardiac pathophysiological processes [2-4]. Continued and deeper understanding of the molecular mechanisms of exosomes in CVDs may help develop promising therapeutic options for these diseases.

The potential therapeutic strategies of exosomes in CVDs became evident in recent years. This special issue entitled "Exosomes in cardiovascular diseases and treatment: experimental and clinical aspects" provides a snapshot in this area. The 9 selected papers on this special topic are dedicated to enhance the current understanding of exosomes in CVDs and will also expand our knowledge on novel clinical treatment.

In this issue, the functions of exosomes in myocardial infarction (MI) were described by 3 different groups. Transplantation of human cardiac progenitor cells (hCPCs) resulted in beneficial effects on the heart after MI. Maring et al. [2] compared the contribution of exosomes by transplanting hCPCs with normal versus reduced exosome secretion. They found that the transplanted hCPCs with low exosome secretion failed to reduce the infarct size post MI. Moreover, injection of hCPCs with normal exosome secretion did significantly reduce the infarct size. Their study suggested

Associate Editor Enrique Lara-Pezzi oversaw the review of this article

Junjie Xiao

junjiexiao@live.cn

1 Cardiac Regeneration and Ageing Laboratory, Institute of Cardiovascular Sciences, School of Life Science, Shanghai University, Shanghai 200444, China that exosome secretion is the driving force behind the shortterm beneficial effect of hCPC transplantation on cardiac recovery after MI. Li et al. [3] summarized the exosomes secreted by different cardiac cells and their functions in cardiac intercellular communications. Also, they discussed the role of these exosomes in cardiac repair after MI. Pan et al. [4] gave an overview on the function of exosomes in the modulation of inflammation and immune response after MI. They also described the immunomodulation by exosomes derived from stem and progenitor cells in the treatment of MI.

Rat H9C2 cardiomyocytes were used to describe the function of exosomes by Li et al. and Liu et al. [5, 6]. Li et al. [5] discussed the role and mechanisms of circulating exosomes in the apoptosis of $\mathrm{H} 9 \mathrm{C} 2$ cardiomyocytes. They found that exosomes attenuated hydrogen peroxide $\left(\mathrm{H}_{2} \mathrm{O}_{2}\right)$-induced apoptosis and improved survival of $\mathrm{H} 9 \mathrm{C} 2$ cells via activation of ERK1/2 signaling pathway. Liu et al. [6] investigated the role of exosomes in doxorubicin (DOX)-induced cardiomyocyte senescence. They proved that miR-34a mediated DOXinduced H9C2 cell senescence by targeting phosphatase 1 nuclear targeting subunit (PNUTS). Moreover, human serum exosomes retarded DOX-induced H9C2 cell senescence by suppressing miR-34a expression. Both of these papers indicated that exosomes might be a potential therapeutic strategy for myocardial injury and cardiac aging.

The cardioprotective mechanisms of stem cells have become a research focus. Ni et al. [7] discussed the current knowledge of stem cells and stem cell-derived exosomes in the cardiovascular system in both health and diseases. Also, they introduced the latest advance in the exosome fields, including the application of exosomes for intracellular delivery of chemical substance, the use of exosomes as biomarkers for diseases, and the critical role of exosomes in regenerative medicine. The potential clinical application of exosomes in diabetic cardiomyopathy (DCM) and atherosclerosis were reported by Tao et al. and Lu et al. [8, 9]. Tao et al. [8] summarized the regulation and function of exosomes in DCM and also highlighted exosomes as potential therapeutic strategies 
for DCM and discussed future research directions for this fastdeveloping field. Lu et al. [9] discussed the potential role of exosome-derived miRNA, protein, and DNA as biomarkers in atherosclerosis pathogenesis, diagnosis, and therapy.

Finally, using a next-generation sequencing (NGS) method, Liu et al. [10] defined the miRNA expression profile of plasma exosomes in spontaneously hypertensive rats (SHR) and normotensive Wistar-Kyoto rats (WKY). Their study detected 27 miRNAs that were differentially expressed between SHR and WKY exosomes and identified hypertensionspecific target genes/signaling pathways. Their finding indicated the selective packaging of miRNAs into exosomes under hypertensive status, which may facilitate the development of potential targets for the diagnosis, prevention, and treatment of hypertension.

In conclusion, this special issue offers a detailed and updated summary about exosomes in CVDs and the potential for treatment from experimental and clinical aspects. Although conventional treatments are available for common cardiovascular problems, exosome therapy becomes a potential treatment option for CVDs with promising future.

Funding Our work was supported by the grants from National Natural Science Foundation of China (81722008, 91639101, and 81570362 to JJ Xiao), the Innovation Program of Shanghai Municipal Education Commission (2017-01-07-00-09-E00042 to JJ Xiao), the grant from Science and Technology Commission of Shanghai Municipality (17010500100 and 18410722200 to JJ Xiao), and the development fund for Shanghai talents (to JJ Xiao).

\section{Compliance with Ethical Standards}

Conflict of Interest The authors declare that they have no conflict of interest.

Research Involving Human Participants and/or Animals This article does not contain any studies with human participants or animals performed by any of the authors.

Informed Consent This article does not contain any studies with human participants.

Publisher's Note Springer Nature remains neutral with regard to jurisdictional claims in published maps and institutional affiliations.

\section{References}

1. Wang, Z., Su, X., Ashraf, M., Kim, I. M., Weintraub, N. L., Jiang, M., et al. (2018). Regenerative therapy for cardiomyopathies. Journal of Cardiovascular Translational Research. https://doi.org/ 10.1007/s12265-018-9807-z.

2. Maring, J. A., Lodder, K., Mol, E., Verhage, V., Wiesmeijer, K. C., Dingenouts, C. K. E., et al. (2018). Cardiac progenitor cell-derived extracellular vesicles reduce infarct size and associate with increased cardiovascular cell proliferation. Journal of Cardiovascular Translational Research. https://doi.org/10.1007/ s12265-018-9842-9.

3. Li, N., Rochette, L., Wu, Y., \& Rosenblatt-Velin, N. (2018). New insights into the role of exosomes in the heart after myocardial infarction. Journal of Cardiovascular Translational Research. https://doi.org/10.1007/s12265-018-9831-z.

4. Pan, W., Zhu, Y., Meng, X., Zhang, C., Yang, Y., \& Bei, Y. (2018). Immunomodulation by exosomes in myocardial infarction. Journal of Cardiovascular Translational Research. https://doi.org/10.1007/ s12265-018-9836-7.

5. Li, P., Liu, Z., Xie, Y., Gu, H., Dai, Q., Yao, J., et al. (2018). Serum exosomes attenuate $\mathrm{H} 2 \mathrm{O} 2$-induced apoptosis in rat $\mathrm{H} 9 \mathrm{C} 2$ cardiomyocytes via ERK1/2. Journal of Cardiovascular Translational Research. https://doi.org/10.1007/s12265-018-97913.

6. Liu, Y., Liu, Z., Xie, Y., Zhao, C., \& Xu, J. (2018). Serum extracellular vesicles retard $\mathrm{H} 9 \mathrm{C} 2$ cell senescence by suppressing miR34a expression. Journal of Cardiovascular Translational Research. https://doi.org/10.1007/s12265-018-9847-4.

7. Ni, J., Sun, Y., \& Liu, Z. (2018). The potential of stem cells and stem cell-derived exosomes in treating cardiovascular diseases. Journal of Cardiovascular Translational Research. https://doi.org/ 10.1007/s12265-018-9799-8.

8. Tao, L., Shi, J., Yang, X., Yang, L., \& Hua, F. (2018). The exosome: A new player in diabetic cardiomyopathy. Journal of Cardiovascular Translational Research. https://doi.org/10.1007/ s12265-018-9825-x.

9. Lu, M., Yuan, S., Li, S., Li, L., Liu, M., \& Wan, S. (2018). The exosome-derived biomarker in atherosclerosis and its clinical application. Journal of Cardiovascular Translational Research. https:// doi.org/10.1007/s12265-018-9796-y.

10. Liu, X., Yuan, W., Yang, L., Li, J., \& Cai, J. (2018). miRNA profiling of exosomes from spontaneous hypertensive rats using nextgeneration sequencing. Journal of Cardiovascular Translational Research. https://doi.org/10.1007/s12265-017-9784-7. 